\title{
Seismic rotation waves: basic elements of theory and recording
}

\author{
Roman Teisseyre $\left({ }^{1}\right)$, Jerzy Suchcicki $\left({ }^{1}\right)$, Krzysztof P. Teisseyre $\left({ }^{1}\right)$, Jan Wiszniowski $\left({ }^{1}\right)$ \\ and Paolo Palangio $\left({ }^{2}\right)$ \\ ( $\left.{ }^{1}\right)$ Institute of Geophysics, Polish Academy of Sciences, Warszawa, Poland \\ ${ }^{2}{ }^{2}$ Istituto Nazionale di Geofisica e Vulcanologia, Roma, Italy
}

\begin{abstract}
Returning to the old problem of observed rotation effects, we present the recording system and basic elements of the theory related to the rotation field and its association with seismic waves. There can be many different causes leading to observed/recorded rotation effects; we can group them as follows: generation of micro-displacement motion due to asymmetry of source processes and/or due to interaction between seismic body/surface waves and medium structure; interaction between incident seismic waves and objects situated on the ground surface. New recording techniques and advanced theory of deformation in media with defects and internal (e.g., granular) structure make it possible to focus our attention on the first group, related to microdisplacement motion recording, which includes both rotation and twist motions. Surface rotations and twists caused directly by the action of emerging seismic waves on some objects situated on the ground surface are considered here only in the historical aspects of the problem. We present some examples of experimental results related to recording of rotation and twist components at the Ojcow Observatory, Poland, and L'Aquila Observatory, Italy, and we discuss some prospects for further research.
\end{abstract}

Key words rotation seismograph - asymmetric stresses - defect density - self-rotation nuclei-rotation and twist motions

\section{Introduction}

Rotation effects at the ground surface associated with earthquakes have been described in several classical monographs, for instance Hobbs (1907) and Davison (1927); the cited examples concern, among other things, twisting of some obelisks, stone graves and segments of columns. Gutenberg (1927) remarked that although such effects are observed, they cannot be explained as being caused by rotation waves, because such

Mailing address: Dr. Roman Teisseyre, Institute of Geophysics, Polish Academy of Sciences, ul. Ks. Janusza 64, 01-452 Warszawa, Poland; e-mail: rt@igf.edu.pl waves do not appear in the ideal elastic theory. Imamura (1937) explained the rotation effects of some objects on the ground surface by the impact of body/surface waves; due to such impact, an object can be inclined, partly losing contact with the ground surface, and when returning to the vertical some twist occurs with respect to its former position. This explanation relates either to differences in the object inertia properties or to differences in cohesion/friction between some parts of the object and the ground surface.

Teisseyre $(1973,1974)$ attributed the appearance of rotation component in seismic waves to coupling between the seismic waves and the micromorphic response of the medium characterized by an internal/granular structure. Droste and Teisseyre (1976) derived the rotation seismogram from the azimuth recording system (the system used consisted of 6 horizontal seismographs oriented in 6 directions differing by 30 degrees) at a site very close (1-3 km) to mine 
event hypocenters (Droste and Teisseyre, 1976; Teisseyre, 1974; reproduced in Teisseyre, 1995, p. 625).

In the last few years, seismologists started to record rotational effects using different types of instruments and systems. The ring coil laser interferometer, based on the Sagnac effect (gyroscope with zero inertia moment), seems to be the most advanced (Takeo and Ito, 1997; Jaroszewicz et al., 2001; Cochard et al., 2003), but some problems arise with application of such gyroscopes to the extremely high sensitivity required in seismology. The rotationseismograph system consisting of two oppositely oriented seismographs has been constructed by Moriya and Marumo (1998); see also Moriya and Teisseyre (1999). However, in our present study we refer to the rotation-seismograph system consisting of two oppositely oriented seismographs, having pendulums suspended on a common axis. Such a seismograph system can record rotation, twist and stretching effects. Twist effects, explained further on, are related to non-uniform rotation, or more exactly to the difference in the rotation effects observed along perpendicular directions (on a plane perpendicular to the axis of rotation).

The question of the proper (self or internal) rotation field - different from the rotation of displacements - may be related to the internal structure of the medium, non-symmetric processes of fracturing, and friction stresses. Shimbo (1975, 1995) and Shimbo and Kawaguchi (1976) have considered the asymmetric stress field related to friction processes and rotation of grains; their conclusion for small deformation fields is that the antisymmetric part of stresses is proportional to the proper rotation tensor, which is independent of the rotation of displacements $(\operatorname{rot} \boldsymbol{u})$. The theoretical basis for rotation and twist deformations in elastic continua with defects and rotation nuclei is outlined by Teisseyre (2002) and by Teisseyre and Boratyński (2002).

For a given shear stress condition, the rotations of grains due to friction are opposite along perpendicular planes (fig. 1a). Usually, however, the earthquake process proceeds along one slip/fracture plane, the main fault, which produces the asymmetry of rotation and friction stress (fig. 1b).

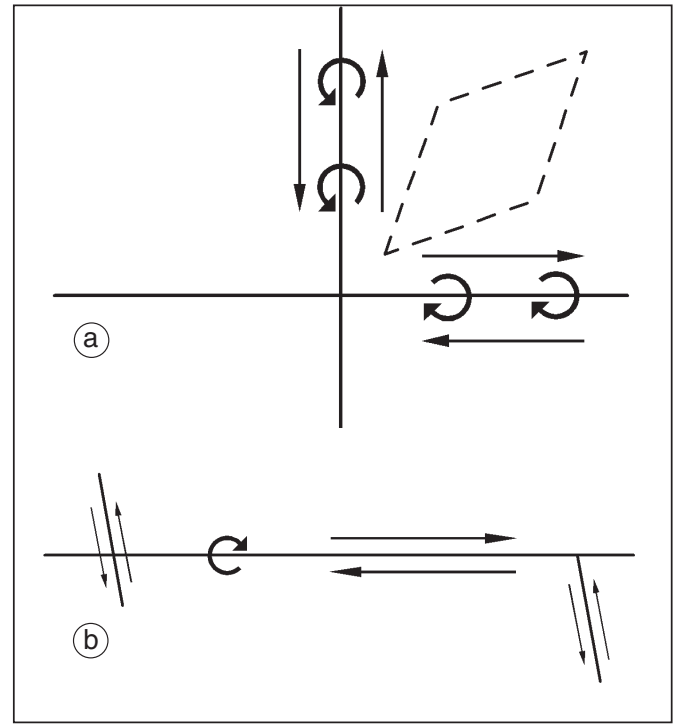

Fig. 1a,b. Fracture planes and rotation effects due to friction processes or dislocation motions: a) the symmetric case; b) the fracture along a main fault; the arrows indicate the shear stresses acting along the fracture planes.

We will not enter here into the theories of continua with internal structure and of continua containing a dense distribution of internal nuclei (dislocations, disclinations, vacancies, thermal nuclei, or electric nuclei) which may be the objects/sources creating internal/self stresses. Some of these internal sources, like dislocations, disclinations and rotation nuclei (rotating grains) cause deviation from stress and strain symmetry; for reference see e.g., Anthony et al. (1968), Kleman (1980), Kossecka and DeWitt (1977) and Teisseyre (2002).

In this paper, we consider theory and experimental results related to micro-displacement motions - rotation and twist - caused by deviations of the real medium from ideal elastic configuration and state. We shall note in advance that the observed rotation and twist effects as recognized by special recording system bring very small motions in the range of $10^{-8} \mathrm{rad} / \mathrm{s}$; it remains an open question whether such motions can cause the macro-rotation effects as experienced by some surface structures after strong earthquakes. 


\section{Rotations as sources of internal stresses}

When considering an elastic continuum with dense distribution of defects (dislocations, disclinations, vacancies, thermal or piezoelectric nuclei and others), or structured continua (e.g., micromorphic, micropolar continua or other media with internal structures), we should take into account the effects of proper rotations expressed in the records of micro-displacement velocity fields. Such fields can arise due to the motion of some defects or small grains forming the continuum.

In earthquake processes, such motions can appear due to relative motions along dislocation planes (medium continuity preserved) or crack surfaces (fracturing process). Internal rotation nuclei can thus be related to dislocation motion (resistance stresses causing rotations of grains) or to slip motion (rotations of grains due to friction stresses along the fracture plane). For symmetric stresses, the rotations along perpendicular planes (fig. 1a) have an opposite sense; the dislocations situated along these perpendicular lines contribute to opposite rotations on those planes. However, earthquake phenomena appear due to motion and fracturing along one main fault (fig. 1b), hence there arises an asymmetry of self stresses and micro-displacement motion linked to these rotation nuclei. We may expect, as discussed later, that these asymmetry effects can only be recorded in the near field.

\section{General approach to real media}

In ideal elasticity, the deformation rates, for a given boundary or initial conditions, can be described by the displacement motions. When approaching a real medium we should also take into account the internal strains or stresses (stress/strain nuclei) related to defects (vacancies, dislocations, disclinations, etc.), structures (plastic, micromorphic, micropolar, etc.) and interaction of these elements with other fields (e.g., thermal, electric, magnetic). To the grain structure, the rotation of grains (rotation nuclei) caused by resistance/friction stresses is added (Shimbo, 1975; Teisseyre, 2002).

Considering theories of continua with defects (see e.g., Kossecka and DeWitt, 1977; Kröner,
1981) or those with micro-structures (see e.g., Eringen and Suhubi, 1964), we shall be aware of the fact that structures of such theories, at least on the level of kinematics, are independent of the length parameter related, e.g., to dimension of grains. Similarly, the observations of motions related to micro-displacement fields do not bring information on the size of grains or defects typical for geological strata or rocks involved.

Following the Kröner approach (1981), we can assume that the deviations from ideal elasticity - due to the above-mentioned factors - can be taken into account by introducing the self (internal) fields. This assures the decomposition of the total field (corresponding to the state of ideal elasticity, expressed by the displacement vector only) into the sum of the self-field and its elastic response. For strain and rotation motions (total, elastic and self parts) we can write

$$
\begin{aligned}
& E^{T}=E^{S}+E, \quad E_{i k}^{T}=\frac{1}{2}\left(\frac{\partial u_{k}}{\partial x_{i}}+\frac{\partial u_{i}}{\partial x_{k}}\right) \\
& \omega^{T}=\omega^{S}+\omega, \quad \omega_{i k}^{T}=\frac{1}{2}\left[\frac{\partial u_{k}}{\partial x_{i}}-\frac{\partial u_{i}}{\partial x_{k}}\right] .
\end{aligned}
$$

In these expressions, the terms on the left-hand sides represent the total field reflecting the ideal elastic state, the next terms, the self-fields, represent internal sources which together with boundary/initial conditions lead to the elastic responses represented by the last terms. In this approach, we can allow the stress-strain relation to remain that established for ideal elasticity. Therefore, similarly to (3.1) we have

$$
S^{T}=S^{S}+S .
$$

The total fields as given by the sum of elastic and self fields should fulfill the compatibility (integrability) conditions in order to be expressed by displacement derivatives. Relations (3.1) and (3.2) can be expressed by the distortion fields defined as follows:

$$
\beta^{T}(u)=E^{T}(u)+\omega^{T}(u), \quad \beta_{i k}^{T}=\frac{\partial u_{k}}{\partial x_{i}}=\beta_{i k}+\beta_{i k}^{S} .
$$


The effects related to deviations from ideal elasticity depend on the real material properties and the thermodynamical state of a body; for rigid brittle rocks under normal conditions such deviations produced by the self/internal distortion field can be very small. However, to study these effects we shall consider the elastic distortion field as it is an observable quantity; from relation (3.4) we obtain

$$
\beta_{i k}=\beta_{i k}^{T}-\beta_{i k}^{S}, \quad \beta_{i k}=\frac{\partial u_{k}}{\partial x_{i}}-\beta_{i k}^{S} .
$$

In the next sections we will explain how to measure the elastic distortion fields.

\section{Rotation seismograph system}

The rotation seismograph system consists of two seismograph sensors situated in parallel, one after the other, with the distance $L$ between the suspension axes, and with the pendulums of opposite orientation. In the system designed at the Institute of Geophysics, Polish Academy of Sciences, the rotation axis of the pendulums is the same $(L=0)$ and pendulum proper length is $l=0.083 \mathrm{~m}$ (fig. 2a,b). A single pendulum seismograph records the displacement velocity $u$ and the displacement velocity derivative (usually neglected as small in comparison to the displacement) multiplied by the pendulum proper length: $l \cdot \partial u / \partial y$, and, similarly, the proper rotation $l \gamma$ (if it exists); in our case $l=0.083 \mathrm{~m}$. With the two opposite seismograph systems we simultaneously record the two fields

$$
u^{\prime}=u+l\left(\frac{\partial u}{\partial y}+\gamma\right), u^{\prime \prime}=u-l\left(\frac{\partial u}{\partial y}+\gamma\right)
$$

It is difficult to separate the terms $\partial u / \partial y$ and $\gamma$; hence, it is convenient to define the micro-displacement velocity tensor as a sum of the derivative of displacement velocity and the appropriate component of the tensor of real rotation

$$
\phi_{i k}=\frac{\partial u_{i}}{\partial x_{k}}+\gamma_{i k}
$$

Thus, the micro-displacement velocity tensor is related to the difference of records between the two opposite sensors (eq. (4.1)) divided by $2 l$

$$
\phi_{i k}=\frac{\Delta u_{i}}{2 l}=\frac{\partial u_{i}}{\partial x_{k}}+\gamma_{i k} .
$$

It is to be noted that our notion of the microdisplacement velocity tensor is different from that of the micro-displacement tensor used in the micromorphic or micropolar continuum (Anthony et al., 1968; Teisseyre and Nagahama, 1999; Nagahama and Teisseyre, 2001); our present definition is related directly to the observables.

Relations (4.1) and (4.3) remain valid, however, only when both seismographs forming the system have exactly the same response characteristics. In their system, Moriya and Marumo (1998) used over-damped sensors; the equalization procedure is, in this case, sufficient to derive the rotation motion. However, for any rotation seismograph we should consider the influence of differences between the response characteristics. We rewrite eqs. (4.1) and (4.3) in the form of the appropriate convolutions

$$
u^{\prime}=U * R^{\prime}+l \Phi * R^{\prime}, \quad u^{\prime \prime}=U * R^{\prime \prime}-l \Phi * R^{\prime \prime}
$$

where we have introduced ground displacement velocity $U$, the response characteristics of both sensors, $R^{\prime}, R^{\prime \prime}$, as well as their mean, $R$, and their difference value, $\Delta R$, and also the rotation ground motion, $\Phi$.

For the difference, $\Delta u$, we write

$$
\Delta u=U * \Delta R+2 l \Phi * R
$$

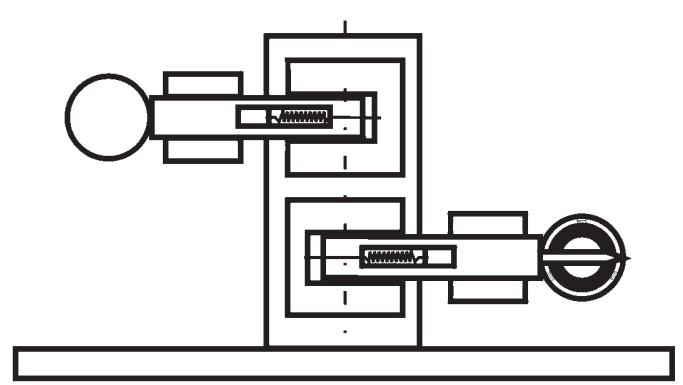

Fig. 2a. Rotation seismograph: scheme. 


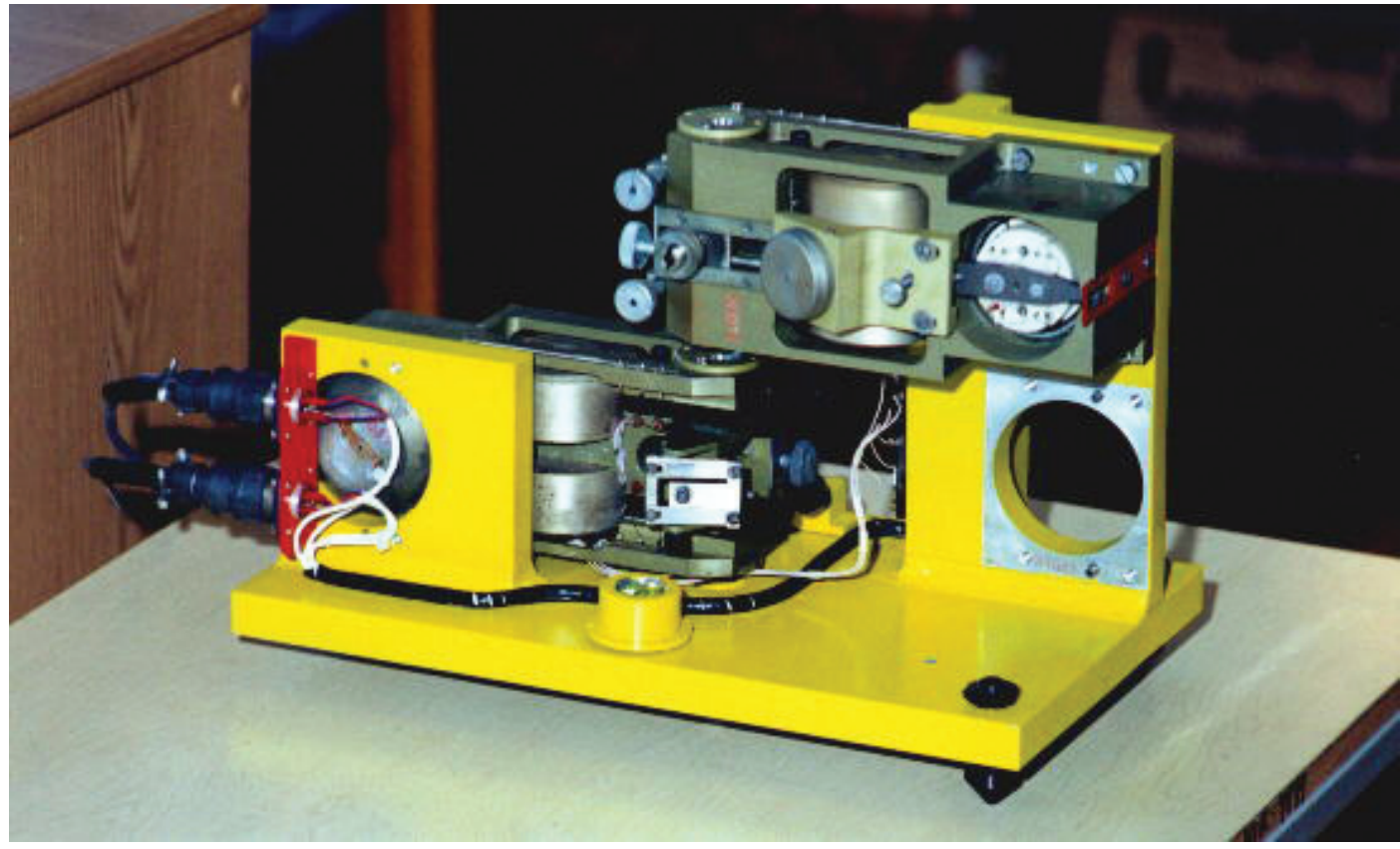

Fig. 2b. Rotation seismograph: photo.

and for the sum of such records we have

$$
\Sigma u=2 U * R+l \Phi * \Delta R \approx 2 U * R
$$

where we neglect the product of two relatively small quantities, namely the product of rotation motion and difference of response characteristics.

To derive the rotation motion, we should minimize the difference between the responses of both sensors, or eliminate such a difference in another way.

\section{Comparison of rotation seismograph sensors}

Even for very similar sensors, the response characteristics of the sensors are slightly different. In our rotation seismograph system, for the aim of comparing both sensors, we can rotate the position of one seismograph sensor in such a way that both the seismograph pendulums, suspended on the common axis, become oriented in the same direction, one just above the other - this is the test position. The working and test positions for the case of the horizontal seismographs are schematically shown in fig. $3 \mathrm{a}, \mathrm{b}$. The records obtained in the test position

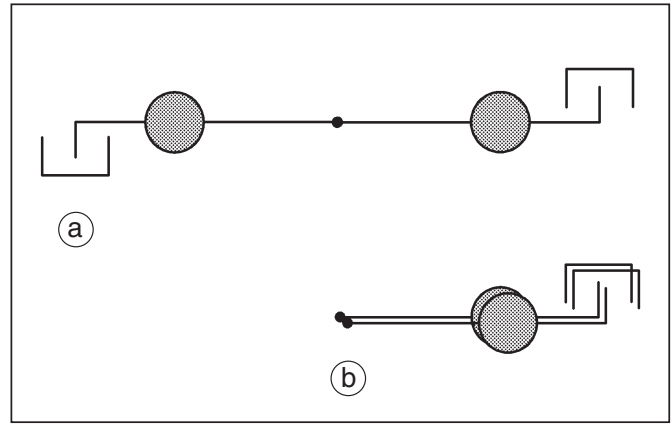

Fig. 3a,b. Rotation seismograph: working (a) and calibration (b) positions of the sensor pendulums. 
could mainly differ due to differences in their response characteristics

$$
u^{\prime}=\left(U^{\prime}+l \Phi\right) * R^{\prime}, \quad u^{\prime \prime}=\left(U^{\prime \prime}+l \Phi\right) * R^{\prime \prime}
$$

and

$$
\Delta u=u^{\prime}-u^{\prime \prime}=(U+l \Phi) * \Delta R \approx U * \Delta R .
$$

To minimize these errors, we can use some numerical procedures based on filtering in frequency or in time domains (Teisseyre et al., 2003; Nowożyński and K.P. Teisseyre, 2003). The respective filters can then be applied to records in the normal working position to reduce the influence of response difference $\Delta R$ disturbing the corresponding difference of records in the working position (eq. (4.4)).

We should underline that our recorded data of displacement velocities are transformed, usually before any analysis, to ground velocities; in such a situation $u^{\prime}$ and $u^{\prime \prime}$ are very close to real ground velocity $U$, and $R^{\prime}$ and $R^{\prime \prime}$ can be treated as corrections to the response characteristics. These characteristics are, thus, very close to unity and $\Delta R$ is much less than unity. However, all these corrections remain unknown; our aim will be to eliminate their influence and to estimate the reliability of micro-displacement motion records.

In the present paper, we use a new method to discern the rotation motion and to estimate the influence of differences in response characteristics of the anti-parallel sensors of our rotation seismograph. Considering the difference of the records $u^{\prime}$ and $u^{\prime \prime}$ in the working position of sensors (eq. (4.5)), $\Delta u=U * \Delta R+2 l \Phi * R$, we should note that it is quite difficult to separate these two terms and separately estimate their mean square values. However, when dividing the FFT spectra of the difference by the sum of records (eqs. (4.5) and (4.6)) we obtain

$$
\begin{gathered}
F(J)=\frac{F(U) F(\Delta R)+2 l F(\Phi) F(R)}{2 F(U) F(R)}= \\
=\frac{F(\Delta R)}{2 F(R)}+\frac{l F(\Phi)}{F(U)}
\end{gathered}
$$

where $F(J)$ is a complex expression in the frequency domain and $J$ is its counterpart in the time domain. We should note that the first term of expression (5.2) depends only on the sensor characteristics, while the second term on input ground motions. This expression may also lead to many outliers whenever the inequality $F(U)<<l F(\Phi)$ occurs. However, we can replace all the outliers with the appropriate mean values plus/minus standard deviations. In our program procedures we divide, at first, the event record into a number of equal segments, that is, into $N$ equal time intervals; then, for each segment $i$ we compute the corresponding complex expressions $F(J(i))$ and afterwards we take the mean value

$$
\begin{aligned}
& F(\bar{J})=\frac{1}{N} \sum_{i=1}^{N} F(J(i)) \\
& F(\bar{J})=\frac{F(\Delta R)}{2 F(R)}+\frac{1}{N} \sum_{i=1}^{N} \frac{l F(\Phi(i))}{F(U(i))} \approx \frac{F(\Delta R)}{2 F(R)}
\end{aligned}
$$

where the approximation is justified only for a big enough number of segments $N$ and provided that the outliers are eliminated.

$$
\text { The obtained value } F(\bar{J}) \approx \frac{F(\bar{\Delta} R)}{2 F(R)} \text { can be }
$$
easily interpolated to the total data number of the record, and then we can consider the following product and difference (compare eqs. (4.5) and (4.6))

$$
\begin{gathered}
F(\bar{J}) F(\Sigma u) \approx F(U) F(\bar{\Delta} R), \\
F(\Delta u)-F(\bar{J}) F(\Sigma u) \approx 2 l F(\Phi) F(R) .
\end{gathered}
$$

From the last expression we can return to the time domain, obtaining the corrected values of micro-displacement motions $2 l \bar{\phi}$ for a given rotation-seismograph.

To illustrate the application of this method we refer to the rotation seismograph records at L'Aquila Observatory, Apennines, of June 16, 2002. In fig. 4 we present the way in which we reach an approximation related to eq. (5.4) (in order to simplify the presentation we have plotted the absolute values of $F(J(i))$ for selected 


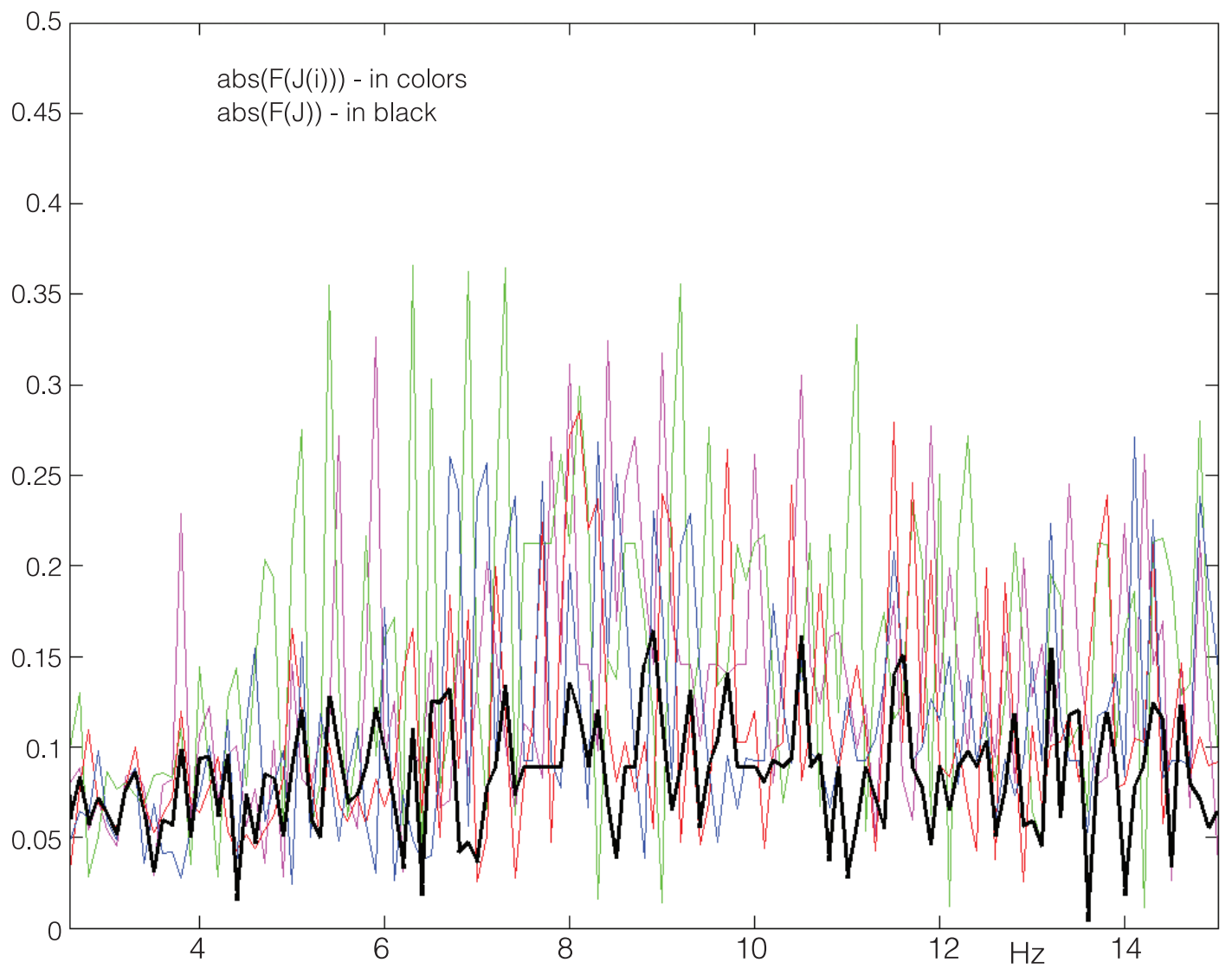

Fig. 4. Examples of the absolute values of the $F J(i)$ functions in the frequency domain computed for the selected time segments (color lines) and the computed mean values (black line) representing function $F J$ (according to the recordings at L'Aquila Observatory of 16 June 2002 event in the Apennines ( $22 \mathrm{~h} 23 \mathrm{~min} ; M=4.1$ ).

segments - color curves and the absolute value of the mean $F(\bar{J}) \approx \frac{F(\bar{\Delta} R)}{2 F(R)}$-black curve). From these estimations we can compute for each time moment the values of recorded rotations $2 l \Phi * R$ and related errors $U * \Delta R$. Figure 5 presents the original plot of difference $\Delta u$ and its parts $2 l \Phi * R$ and $U * \Delta R$, proving the successful result of the method applied. Then we calculated the ratio of the mean square values of recorded rotations and the mean square values of displacement velocities, mean $\left(2 l \Phi^{*} R\right)^{2} /$ mean $u^{2}$, and the ratio of the mean square values of respective errors and the mean square values of displacement velocities, mean $(U * \Delta R)^{2} /$ mean $u^{2}$; for the case under consideration, the first ratio amounts to 0.012 , while the second is much smaller at 0.0014 .

This analysis proves that the recorded microdisplacement motions, as derived from differences of two anti-parallel sensors, exceed the estimated errors due to calibration uncertainties and other system defects.

\section{Recording the twist and rotation motions: examples}

To record the components of the microdisplacement velocity $\phi_{i k}$ (rotation tensor $\gamma_{i k}$ plus 


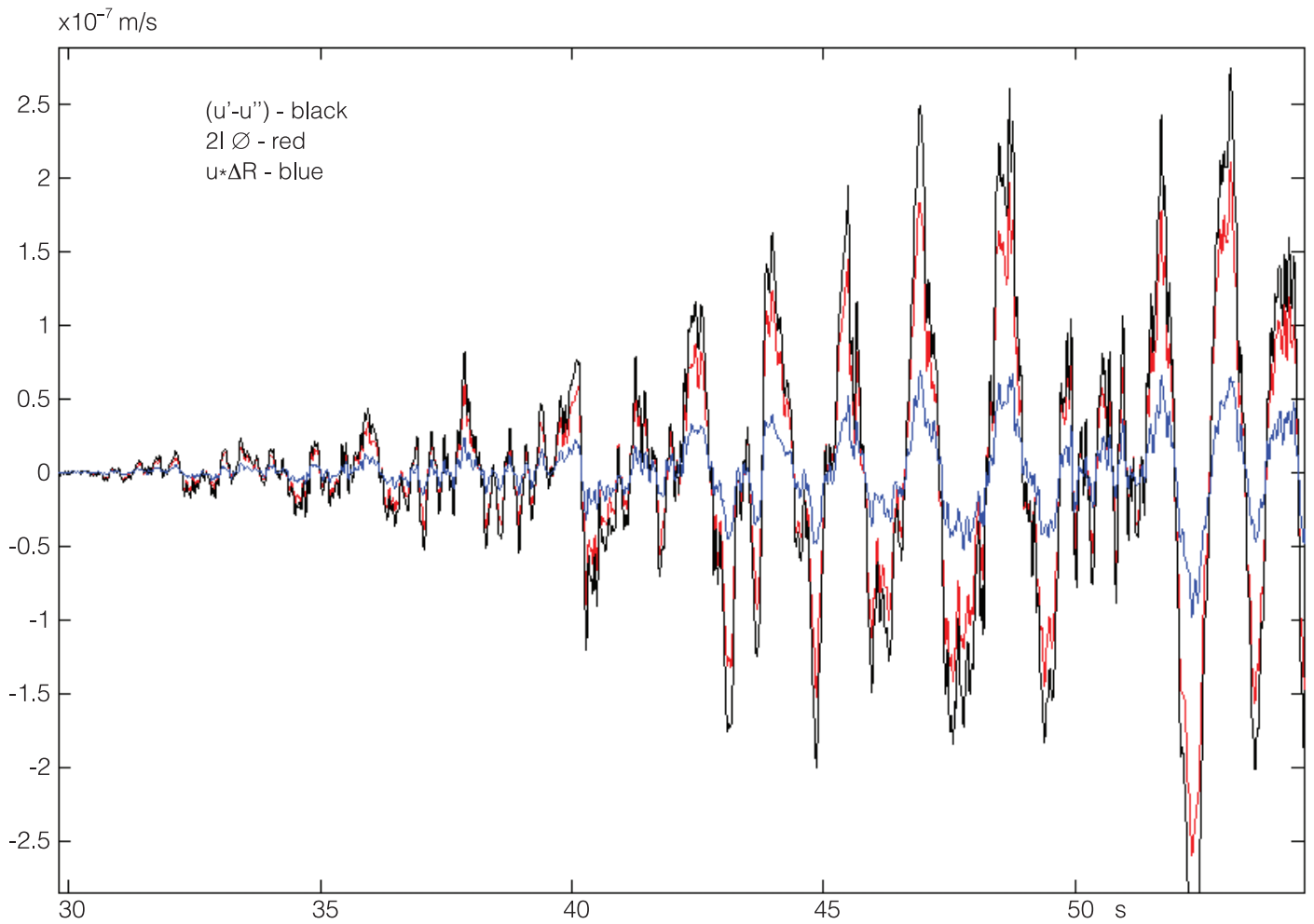

Fig. 5. Micro-displacement velocity, i.e. the difference between the displacement velocities recorded by the two anti-parallel sensors (black curve) and the corrected micro-displacement field (red curve) and the computed errors due to response difference (blue curve).

rotation of displacements: eq. (4.3)), we shall use three rotation seismograph systems: each one with two oppositely oriented pendulums and sensors for each axis of rotation, and another three seismograph systems, each one consisting of the pair of the oppositely oriented coil sensors, to record the velocities of stretching components $\phi_{11}, \phi_{22}, \phi_{33}$.

For the case of vertical axis, we use a pair of two rotation systems as shown in fig. 6 (note that the construction of our rotation seismograph is such that the upper sensor coincides with the negative derivative $-\partial u / \partial y$; for this reason, the component $u$ should be taken with the «minus» sign to obtain the proper definition of the micro-displacement tensor components as given in eq. (4.2)). For this system, we will use the following notations for displacement and micro-displacement velocities

$$
u=u_{1}, \quad v=u_{2}, \quad \phi=\phi_{12}, \quad \psi=\phi_{21} .
$$

Thus, for these two rotation seismograph systems, we shall use, in addition to eq. (4.1) for the first system, a similar relation for the perpendicularly situated second rotation-seismograph system

$$
v^{\prime}=v+l\left(\frac{\partial v}{\partial x}+\gamma^{\prime}\right), v^{\prime \prime}=v-l\left(\frac{\partial v}{\partial x}+\gamma^{\prime}\right)
$$

The rotation and twist motions are given by the difference and sum of the micro-displacement velocities, respectively 


$$
\begin{aligned}
& R=\frac{1}{2}[\phi-\psi]=\frac{1}{2}\left[\frac{\partial u}{\partial y}-\frac{\partial v}{\partial x}\right]+\frac{1}{2}\left[\gamma_{x y}-\gamma_{y x}\right] \\
& T=\frac{1}{2}(\phi+\psi)=\frac{1}{2}\left(\frac{\partial u}{\partial y}+\frac{\partial v}{\partial x}\right)+\frac{1}{2}\left(\gamma_{x y}+\gamma_{y x}\right) .
\end{aligned}
$$

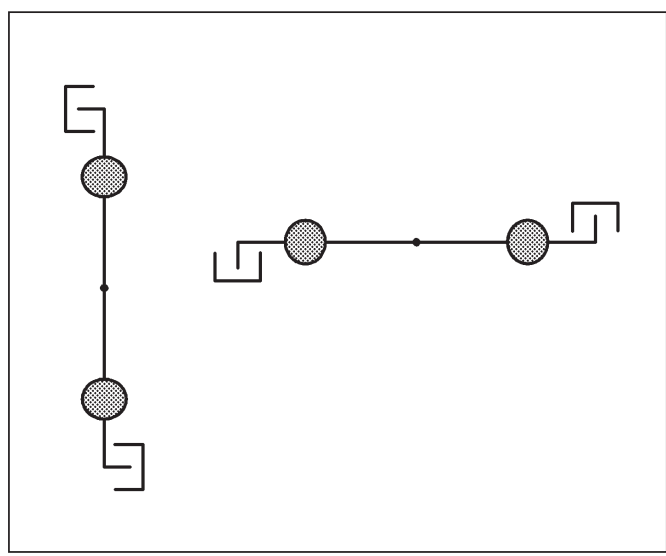

Fig. 6. Scheme of the rotation seismograph system related to one axis of rotation.
Correspondingly, one term contains rotation/ strain motion related to displacement velocities, while the other contains the proper (self) fields of rotation/twist.

The theories of continua with structure and defects predict a complex pattern of microdeformations and motions; the rotation tensor becomes asymmetric (not anti-symmetric as for pure rotation), and we also observe twist deformation (fig. 7).

We can now compare the theoretically derived relation for total distortion $\beta^{T}$ with the definition (4.2); comparing these relations we can write

$$
\frac{\partial u_{k}}{\partial x_{i}}=\beta_{i k}+\beta_{i k}^{S} \approx \phi_{i k}-\gamma_{i k}, \quad \beta_{i k}^{S}=-\gamma_{i k}
$$

where we have assumed that the self-rotations (proper rotations and twists) are the unique sources of self-distortions.

Now, we can write

$$
\beta_{i k}=\phi_{i k}
$$

identifying the observed field with the distortion

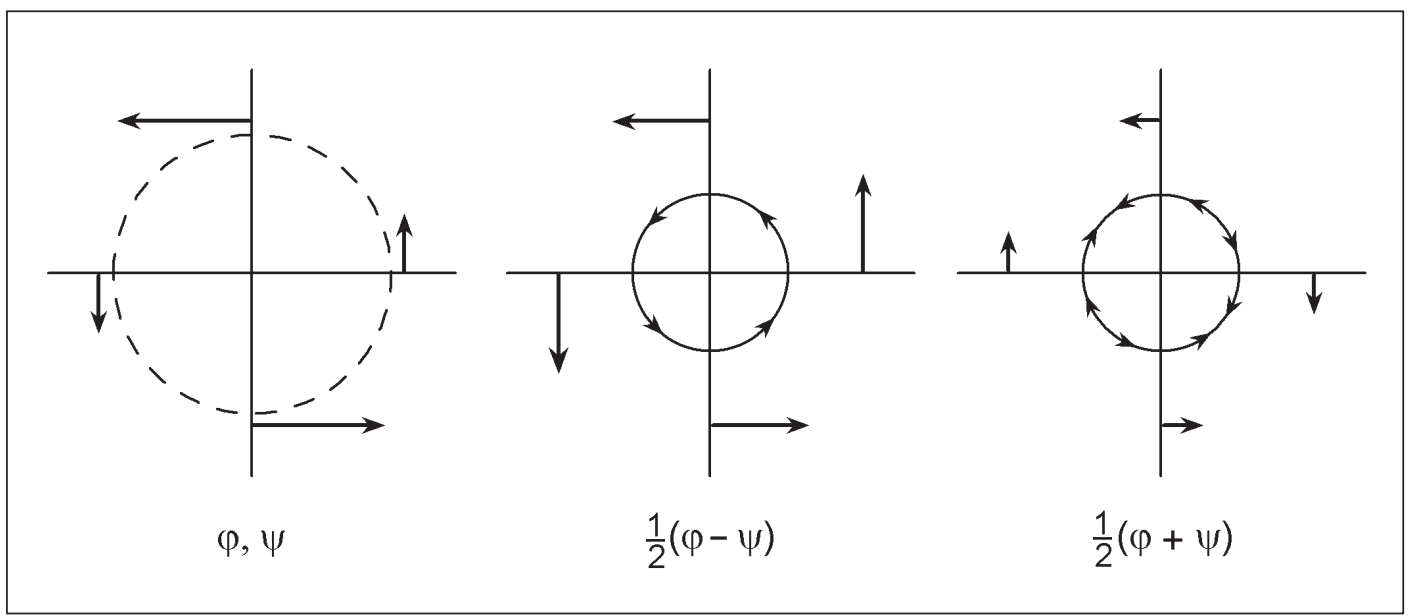

Fig. 7. Scheme presenting the micro-displacement components related to the grain surface (left), the related rotation (center) and twist motion (right). 

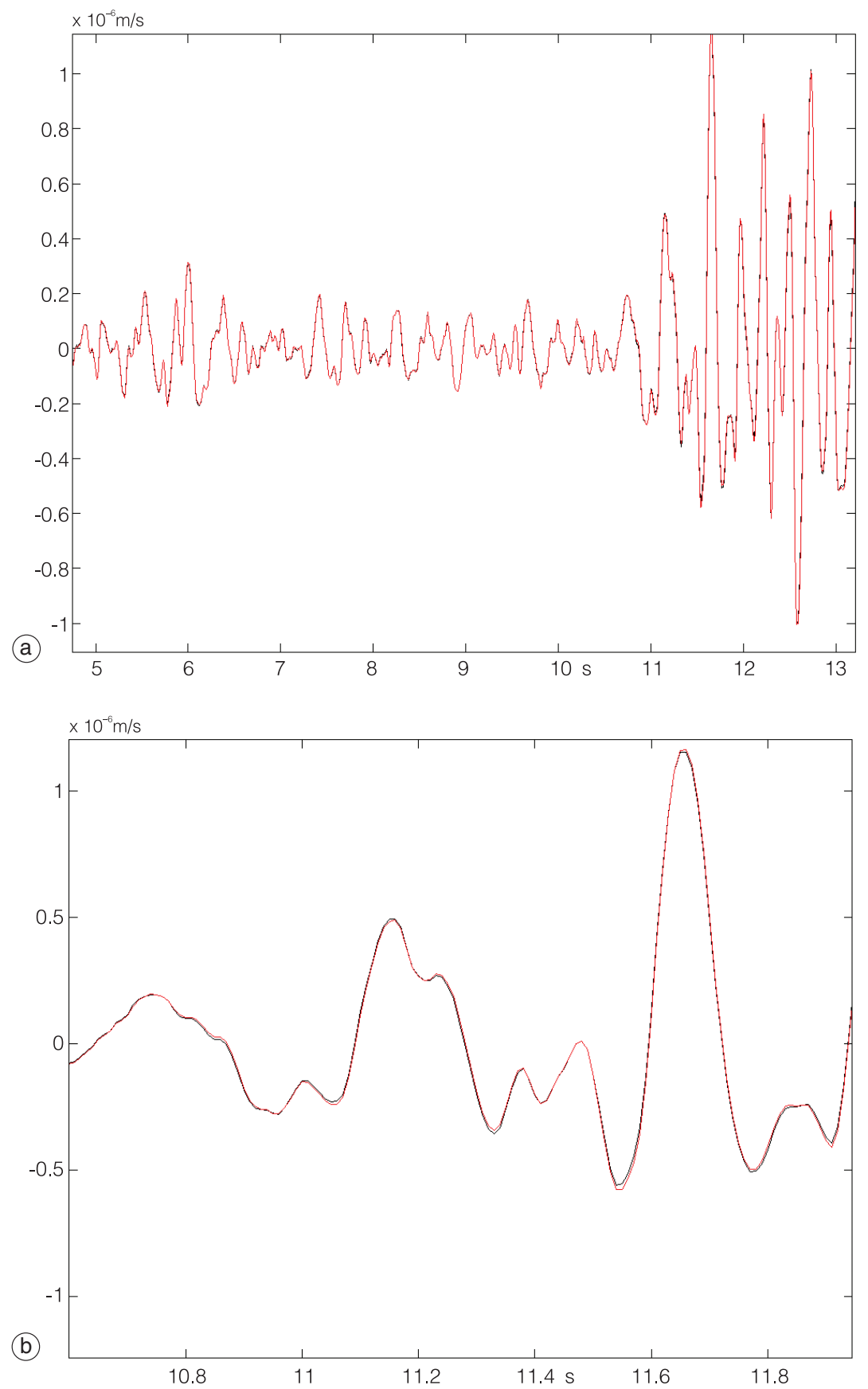

Fig. 8a,b. Example of recording of the displacement velocities by two anti-parallel sensors (Ojcow Observatory, record of a Silesian event at distance about 60 km; 11 July 2001, 18 h 50 min; magnitude about 1.5); parts (a) and (b) are in different time scales. 
field. The self-rotation is expressed by the proper rotation $\gamma_{[i k]}$ and the twist part $\gamma_{(i k)}$, as in eqs. (6.3) and (6.4).

We will now present some records obtained with the use of our system of rotation seismographs at the Ojcow Observatory, Poland, and at the L'Aquila Observatory, Italy.

Figures 8a,b and 9 present data of a Silesian seismic event recorded at Ojcow; the distance from the source is about $60 \mathrm{~km}$ (11 July 2001, $18 \mathrm{~h} 50 \mathrm{~min}$; magnitude about 1.5); fig. 8a,b gives an example of the displacement velocity records $u_{2}^{\prime}$ and $u_{2}^{\prime \prime}$ in $\mathrm{m} / \mathrm{s}$ for two anti-parallel sensors of the rotation seismograph: parts (a) and (b) are in different time scales. Only very small differences can be seen in these records, contributing to the micro-displacement velocity field; fig. 9 presents an example of recordings for the microdisplacement velocity components, $\phi$ and $\psi$, in $\mathrm{rad} / \mathrm{s}$ as obtained from the two perpendicularly situated rotation seismographs. According to relations (4.5) and (4.6) these components make it possible to derive the respective rotation and twist motions. Figure 10 presents the rotation and twist velocities derived from the records at L'Aquila Observatory of the 16th June 2002 seismic event ( 22 h 23 min; $M=4.1$; Apennines); the black curves show original data, whereas the red ones show the values corrected by the method outlined above (see eqs. (5.2)-(5.4)).

\section{Discussion and conclusions}

Among many open questions related to seismic rotation waves, the most important concern their generation mechanism and information contained in such waves. We can
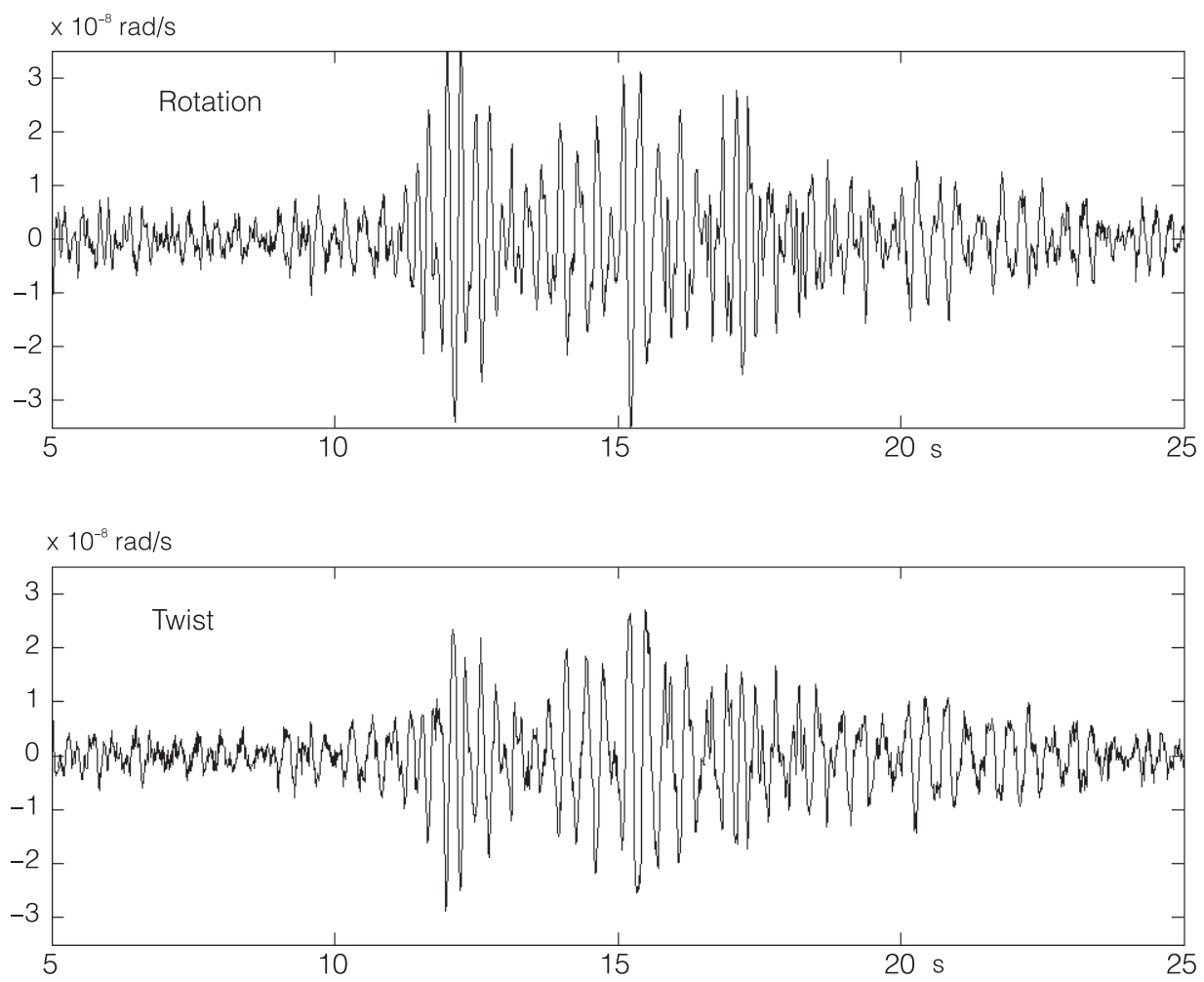

Fig. 9. Records of the rotation and twist velocity components (Ojcow Observatory; as above) 

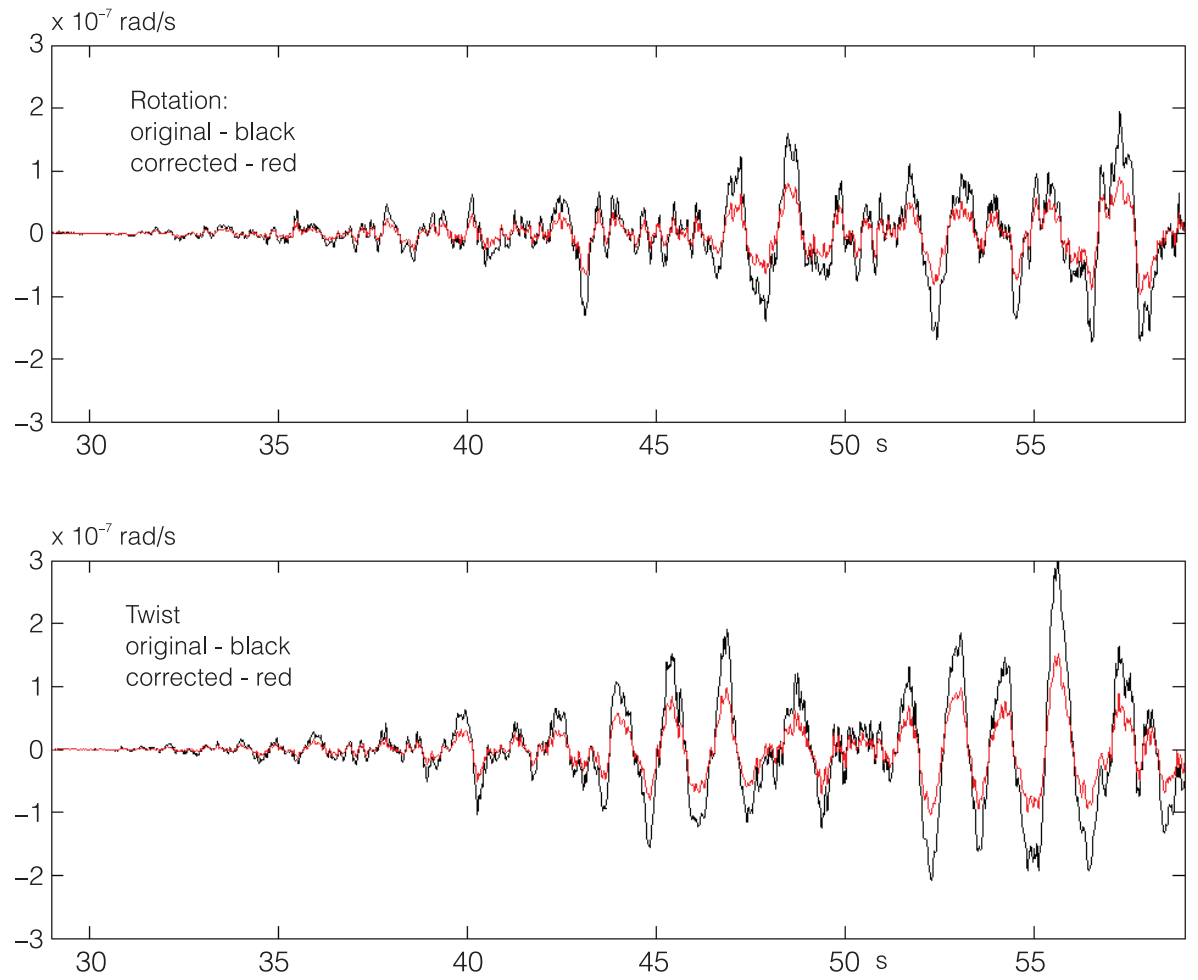

Fig. 10. Rotation and twist velocities derived from the records at L'Aquila Observatory of the 16 June 2002 seismic event in the Apennines; the black curves show the raw data, whereas the red ones show the corrected values.

briefly consider three different, but maybe concurrent, origins of micro-displacement motion. The first relates the micro-displacement field directly to rotation, or generally to space derivatives of displacement. To estimate the order of magnitude of displacement velocity derivatives we can use the plane wave theory and estimation based on direct records

$$
u_{i}=A_{i} \exp \left(i k_{n} x_{n}-i \omega t\right), \frac{\partial u_{i}}{\partial x_{k}} \approx-\frac{1}{V} \frac{\partial u_{i}}{\partial t}
$$

where $V$ is the wave velocity near the surface at the station site and we disregard the direction cosine related to the wave approach direction; a possible anisotropy effect would not exceed a factor of 2 .
The rotation or twist motions can thus be estimated a priori and then compared with the observed data of micro-displacement motions. For the records at the Ojcow Observatory, we should assume an unrealistically small value of wave velocity, of the order of $50 \mathrm{~m} / \mathrm{s}$, to obtain the best fit of the observed micro-displacement motion and the corresponding field estimated from the plane wave theory (fig. 11). The interference of different seismic phases related to body shear and surface waves can contribute to somewhat more realistic results. Also we should note that such an estimation based on ideal elasticity may differ from estimates based on a more realistic assumption for medium properties. Takeo and Ito (1997) have estimated, in a very elegant form, using the differential geometry approach, the values of displacement 
velocity rotation for a medium with dense defect distribution and found, based on the synthetic rotation seismogram at a distance of about $5 \mathrm{~km}$ from the source, that the results of such an estimation can be convincing.

The last remark leads us to the second origin mechanism that can attribute the formation of rotation motions due to interaction between the seismic body and surface waves in a medium with internal structure and defects. Theories of the micropolar and micromorphic media (Eringen and Suhubi, 1964) present a continuum approach to granular media and predict the appearance of such micro-displacement motions; the corresponding wave solutions lead, however, to a proportionality relation between the self micro-displacement motion and the derivative of displacement velocity (Teisseyre and Nagahama, 1999; Nagahama and Teisseyre, 2001), modifying the estimation considered above to a new form

$$
\begin{gathered}
\gamma_{i k}=C \frac{\partial u_{i}}{\partial x_{k}}, \\
\phi_{i k}=(C+1) \frac{\partial u_{i}}{\partial x_{k}} \approx-\frac{C+1}{V} \frac{\partial u_{i}}{\partial t}=-\frac{1}{V^{A}} \frac{\partial u_{i}}{\partial t}
\end{gathered}
$$

where $C$ is the coupling parameter expressing

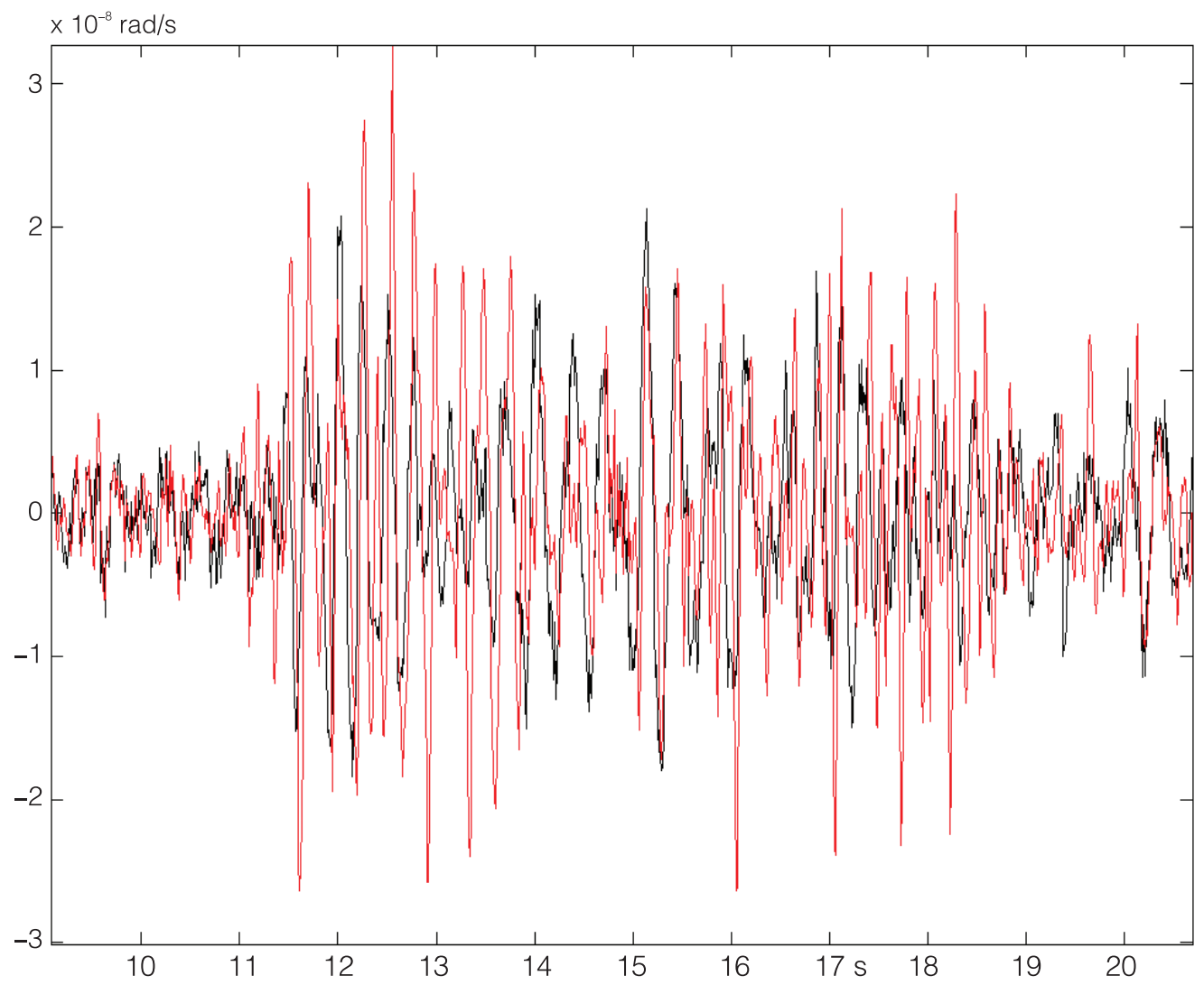

Fig. 11. Comparison between the observed micro-displacement velocity field (black curve) and the estimated derivative of displacement velocity (red curve) with the appropriately chosen apparent wave velocity for the best fit of both curves (here $V=50 \mathrm{~m} / \mathrm{s}$ ); the correlation coefficient equals to 0.5 (Ojcow Observatory; as above). 
interaction of the seismic body and surface waves with the internal structure, defects and any potential rotation nuclei in the medium; this parameter can be in reality considered as a frequency-dependent function appropriate for the medium; $V^{A}=(C+1) / V$ can be defined as the apparent micromorphic wave velocity.

We should note that the micro-displacement motion (4.3) contains two terms, difficult to separate in an experiment, unless a special arrangement of seismometers is applied, for example, three pendulum seismometers arranged in one line, or an array of one pendulum and at least two coil sensors.

Finally, we can consider the seismic source process and its asymmetry pattern as a cause of micro-displacement motions as we mentioned in previous sections of this paper. According to Shimbo $(1975,1995)$, fracture slip motion causes rotations of the grains adjacent to the fracture plane; the anti-symmetric part of selfstresses and self-strains becomes related to the rotation motion

$$
\gamma_{[m n]}=\frac{A}{2 \mu} S_{[m n]}^{S}=-\frac{A}{2 \mu} S_{[m n]}=-E_{[m n]}=E_{[m n]}^{S}
$$

where the new constant $A$ depends on friction (for the fracture slip process) or on stress resistance (for dislocation motion) and the grain inertiamoment properties.

This friction law indicates the appearance of the anti-symmetric self-stress field and has been generalized by Teisseyre (2002) and, Teisseyre and Boratyński (2002) to become the constitutive law joining the anti-symmetric part of strains and stresses. Of course, we should be aware of the fact that the self-rotation motion once generated at a seismic source could be quickly attenuated when propagating through layers with properties not deviating far from those of ideal elasticity; we are close to believing that micro-displacement motion can bear information on source processes only when it is recorded in the near field.

Concluding, we dare say that classical seismology, based on recording the displacement velocities, remains on the level of an ideal elastic continuum, whereas when introducing recordings of the micro-displacement tensor velocities we approach the level of a real continuum.
In further studies, we will encounter many new problems. First of all, we need very sensitive digital and numerically accurate instruments to record all 9 components of the micro-displacement tensor. We will need data at different observation sites and for different earthquakes. We should study many new relations, like that for the mean square ratio between the displacement velocities and rotations and twists as a function of the source-station distance. We should also clarify the influence of the local structure and that of the source process.

\section{Acknowledgements}

The paper has been partly sponsored by the Polish Committee for Scientific Research, Grant No. 6 P04D 06620.

\section{REFERENCES}

Anthony, K., U. Essmann, A. Seeger and H. Truble (1968): Disclinations and the Cosserat-continuum with incompatible rotations, in Mechanics of Generalized Continua, edited by E. KRÖNER (Springer Verlag, Berlin), 355-358.

Cochard, A., A. Flaws, U. Schreiber and H. IGEL (2003): Observations and simulations of rotational motions, Geophys. Res. Abstr., 5, 13160.

DAVISON, CH. (1927): The Founders of Seismology (Cambridge University Press, Cambridge), pp. 240.

Droste, Z. and R. Teisseyre (1976): Rotational and displacement components of ground motion as deduced from data of the azimuth system of seismographs, Publs. Inst. Geophys. Pol. Acad. Sci., 97, 157-167.

ERINGEN, A.C. and E.S. SuHUBI (1964): Nonlinear theory of simple microelastic solids, I, Int. J. Eng. Sci., 2, 189-203.

GuTENBERG, B. (1927): Grundlagen der Erdbebenkunde (Univ. Frankfurt a/M), pp. 189.

HoBBS, W.H. (1907): Earthquakes. An Introduction to Seismic Geology (Appleton and Co., New York), pp.336.

ImAMurA, A. (1937): Theoretical and Applied Seismology (Maruzen Co., Tokyo), pp. 358.

JAROSZEWICZ, L.R., Z. KRAJEWSKI and R. ŚWILLO (2001): Application of fiber-optic Sagnac interferometer for detection of rotational seismic events, Mol. Quantum Acoust., 22, 133-144.

KLEMAN, M. (1980): The general theory of disclinations, in Dislocations of Solids, edited by F.R.N. NABARRO (North-Holland Publ. Comp.), Other Effects of Dislocations: Disclinations, vol. 5, 243-297.

KosSECKA, E. and R. DEWITT (1977): Disclination kinematic, Arch. Mech., 29, 633-651.

KRÖNER, E. (1981): Continuum theory of defects, in Physique 
des Defauts / Physics of Defects, edited by R. BALIAN, M. KLEMAN and J.-P. POIRIER (North Holland Publ. Comp., Dordrecht), Volume Les Houches, Session 35, 215-315.

MoriYA, T. and R. MARUMO (1998): Design for rotation seismometers and their calibration, Geophys. Bull. Hokkaido Univ., 61, 99-106.

MoriyA, T. and R. TEISSEYRE (1999): Seismic rotation waves recorded from the Usu volcanic events, Japan, Acta Geophys. Pol., 47, 351-362.

NAGAHAMA, H. and R. TeISSEYRE (2001): Seismic rotation waves: dislocations and disclinations in a micromorphic continuum, Acta Geophys. Pol., 49, 120-129.

NOWOŻYŃSKI, K. and K.P. TEISSEYRE (2003): Time-domain filtering of seismic rotation waves, Acta Geophys. Pol., 51, 51-61.

SHIMBO, M. (1975): A geometrical formulation of asymmetric features in plasticity, Bull. Fac. Eng. Hokkaido Univ., 77, 155-159.

SHIMBO, M. (1995): Non-Riemannian geometrical approach to deformation and friction, in Theory of Earthquake Premonitory and Fracture Processes, edited by $\mathrm{R}$. TEIsseyre (PWN - Polish Scientific Publishers, Warszawa), 520-528.

Shimbo, M. and M. KAWAGUCHI (1976): A note on the asymmetric fields, Bull. Fac. Eng. Hokkaido Univ., 80, 75-79 (in Japanese).

TAKEO, M. and H.M. ITO (1997): What can be learned from rotational motions excited by earthquakes?, Geophys.
J. Int., 129, 319-329.

TEISSEYRE, R. (1973): Earthquake processes in a micromorphic continuum, Pure Appl. Geophys., 102 (1), 15-28.

TEISSEYRE, R. (1974): Symmetric micromorphic thin space continuum: wave propagation, point source solutions and some applications to earthquake processes, in Continuum Mechanics Aspects of Geodynamics and Rock Fracture Mechanics, edited by P. THOFT-CHRISTENSEN, NATO Advanced Study Institute Series (D. Reidel Publ. Comp., Dodrecht-Holland/Boston U.S.A.), 201-244.

TEISSEYRE, R. (1995): Symmetric micromorphic theory; applications to seismology, in Theory of Earthquake Premonitory and Fracture Processes, edited by R. TeISSEYRE (PWN - Polish Scientific Publishers, Warszawa), 616-627.

Teisseyre, R. (2002): Continuum with defect and self rotation fields, Acta Geophys. Pol., 50, 51-68.

TEISSEYRE, R. and W. BorATYŃSKI (2002): Continuum with self-rotation nuclei: evolution of defect fields and equations of motion, Acta Geophys. Pol., 50, 223-230.

Teisseyre, R. and H. NAgahama (1999): Micro-inertia continuum: rotations and semi-waves, Acta Geophys. Pol., 47, 259-272.

TeISSEYRE, R., J. SUCHCICKI and K.P. TEISSEYRE (2003): Recording the seismic rotation waves: reliability analysis, Acta Geophys. Pol., 51, 37-50.

(received January 20, 2003; accepted May 27, 2003) 